\title{
COMBINING ABILITY AND HETEROSIS FOR SOME FLOWERING AND VEGETATIVE TRAITS OF FIVE MAIZE INBREDS UNDER TWO NITROGEN LEVELS FERTILIZATION
}

Sultan, M. S. ${ }^{1}$; M. A. Abdel-Moneam ${ }^{1}$; S. M. G. Salama ${ }^{2}$ and A. M. EL. Oraby ${ }^{3}$ 1Dept. of Agronomy, Fac. Agric. , Mans. Univ. 2Central Lab. for Design and Statistical Analysis Res. ARC Egypt. 3Fine Seeds International Company.

\begin{abstract}
A half diallel cross among 5 inbred lines of maize was evaluated under two different nitrogen rates for flowering and vegetative characters i.e. anthesis date, silking date, Anthesis-Silking Interval (ASI), plant height, ear height, ear leaf area and stem diameter, to evaluate the role of GCA and SCA of inbred lines in hybrids performance under high and low of nitrogen levels, and to establish the magnitude of heterosis as well as both GCA and SCA effects and their interaction with the nitrogen fertilization rates. Mean squares were significant for all studied traits except, ear leaf area under normal nitrogen level. General and specific combining ability (GCA and SCA) mean squares were significant for all studied traits, except stem diameter under normal nitrogen level at GCA and ear leaf area under normal nitrogen level at GCA and SCA. GCA/SCA ratios revealed that the non-additive gene action for anthesis date, silking date, plant height under normal nitrogen, ASI, ear height, stem diameter under both nitrogen levels and ear leaf area under stress nitrogen level. While, the additive gene action was recorded for anthesis date, silking date and plant height under stress nitrogen level and ear leaf area under normal nitrogen level. The additive gene action was the most expression under normal nitrogen level. The best combiners were $\mathrm{P}_{2}$ (Inb.95) and $\mathrm{P}_{4}$ (Inb.204) inbred lines for most of studied traits under normal and stress nitrogen levels. This result indicated that these inbred lines could be considered as good combiners for improving these traits. The best crosses for anthesis and ASI date was $\mathrm{P}_{2}$ (Inb.95) X $\mathrm{P}_{5}$ (Inb.213), for silking date was $\mathrm{P}_{2}$ (Inb.95) X $\mathrm{P}_{3}$ (Inb.144), for plant height was $P_{3}$ (Inb.144) X $P_{5}$ (Inb.213), ear height was $P_{1}$ (Inb.84) $\mathrm{X} \mathrm{P}_{4}$ (Inb.204) and stem diameter was $\mathrm{P}_{4}$ (Inb.204) X $\mathrm{P}_{5}$ (Inb.213) under both nitrogen levels. These crosses could be selected and used in breeding programs for improving these traits. Results showed significant heterosis for all studied traits. The best crosses over both their mid-parents and better-parents for anthesis date and silking date was $\mathrm{P}_{2}$ (Inb.95) $\times \mathrm{P}_{3}$ (Inb.144) under both nitrogen levels for ASI date was $\mathrm{P}_{2}$ (Inb.95) X $\mathrm{P}_{5}$ (Inb.213), for ear height was $\mathrm{P}_{1}$ (Inb.84) X $\mathrm{P}_{4}$ (Inb.204), for plant height was $P_{3}$ (Inb.144) X $P_{5}$ (Inb.213), for ear leaf area was $P_{1}$ (Inb.84) $\mathrm{X}_{3}$ (Inb.144) under stress nitrogen level and for stem diameter were $\mathrm{P}_{2}$ (Inb.95) X P 5 (Inb.213), P 3 (Inb.144) X P $P_{5}$ (Inb.213) and $P_{4}$ (Inb.204) X $P_{5}$ (Inb.213).
\end{abstract}

\section{INTRODUCTION}

Maize belongs to poaceae family and tribe Maydaeae. Maize is an annual short day, cross pollinated crop. Based on area and production, maize is the $3^{\text {rd }}$ most important cereal crop after wheat and rice, all over 
the world for production and consumption. In addition to its use as a human food, it is also utilized as a poultry and livestock feed. Moreover it is also used for many industrial purposes (White and Johnson, 2003). Maize (Zea mays L.) forms major dietary part of millions of people in the form bread making where the government policy is to mix wheat flour $(80 \%)$ with maize flour (20\%) in bread making all over the country in order to reduce wheat imports. Maize is the most important crop in eastern and southern Africa in area harvested and the contribution of calories and protein to diets (FAO, 2011). Nitrogen is the most important nutritive element for the worldwide production of cereals (wheat, barley, rice, sorghum and maize). It is mostly supplied to the soil in the form of inorganic fertilizers and to a lesser extent as organic manure. A considerable portion of fertilizer $\mathrm{N}$ is lost through gaseous plant emissions, soil denitrification, surface runoff, ammonia volatilization, and leaching (Akintoye et al., 1999 and Raun and Johnson, 1999) These losses account for the low estimate of $33 \%$ for worldwide $\mathrm{N}$-use efficiency (NUE) (NUE=N recovered / $\mathrm{N}$ supplied) with a slightly higher $42 \%$ estimate for developed than developing 29\% countries (Raun and Johnson, 1999) The affordability of $N$ in the developed countries has led to its misuse and over application (Raun and Johnson, 1999) and created growing environmental concerns from increased nitrate leaching that may lead to ground water contamination. In contrast, the rates of $\mathrm{N}$ fertilizers in most developing countries are considerably low because of the limited access to fertilizers and the low purchasing power of small farmers due a high fertilizer/cereal grain price ratio. The two main genetic parameters of diallel analysis are GCA and SCA which are essential in developing breeding strategies. In this concern, several investigators reported that additive gene action was responsible for the inheritance of growth characters (Sedhom, 1994; Ahmed et al, 2000; AlNagger et al, 2002; Alamnie et al, 2006 and El-Badawy, 2006 ). However, Dadheech and Joshi, (2007), Barakat and Osman, (2008) and Irshad-El-Haq et al, (2010) reported that non-additive gene action was more important in the inheritance of agronomic traits in maize. Therefore, the objectives of this study were evaluate the role of GCA and SCA of inbred lines in hybrids performance under high and low of nitrogen levels, and to establish the magnitude of heterosis as well as both GCA and SCA effects under two nitrogen fertilization rates.

\section{MATERIALS AND METHODS}

Plant materials: Five inbred lines of corn (Zea mays, L.) were used as parents in this study i.e., $\mathrm{P}_{1}(\operatorname{Inb} .84), \mathrm{P}_{2}$ (Inb95), $\mathrm{P}_{3}$ (Inb. 144), $\mathrm{P}_{4}$ (Inb. 204) and $P_{5}(\operatorname{Inb} .213)$. These inbred lines were obtained from Agriculture Research Center (ARC).

Field experiments: The field trials were started in the 2011 growing season in the Experimental Farm of the Faculty of Agric., Mansoura Univ., and lasted in 2012. 
In 2011 growing season, the five parental inbred lines were planted on April $21^{\text {st }}$ and May $7^{\text {th }}$, and each inbred line was grown in two rows, to overcome the differences in flowering date and to secure enough hybrid seeds. During this season, all possible cross combinations, without reciprocals, were made giving a total of $10 \mathrm{~F}_{1}$,s hybrid seeds. In 2012 growing season, 16 entries (10 $F_{1}$ 's along their 5 parental inbred lines plus one check cultivar (S.C.10)) were grown in two experiments representing two different nitrogen levels, which were $60 \mathrm{Kg} \mathrm{N} /$ fad (stress) and $120 \mathrm{Kg} \mathrm{N} /$ fad (normal) by using distance of $70 \mathrm{~cm}$ between ridges and $25 \mathrm{~cm}$ between hills. Each experiment was designated in a Randomized Complete Blocks Design (RCBD) with three replicates. Each plot consisted of one ridge three meters long. Hills were thinned after seedling emergence to secure one plant per hill. Each experiment was hoed twice, before first and second irrigations. Phosphorus in the form of calcium super phosphate (15.5\% P2O5) at a rate of $200 \mathrm{~kg} / \mathrm{fad}$, was added to the soil during seedbed preparation, and potassium sulphate $(48 \% \mathrm{~K} 2 \mathrm{O})$ at a level of $50 \mathrm{~kg} / \mathrm{fad}$ was applied after thinning. Moreover, nitrogen in the form of Urea $(46 \% \mathrm{~N})$ at a rate studied $(60$ and $120 \mathrm{~kg} / \mathrm{fad})$ was added in two equal split doses, before the first and the second irrigation. Other agriculture practices were applied as recommended.

Studied traits: The following measurements were recorded: anthesis date (day), silking date (day), anthesis-silking interval (ASI) (day), plant height $(\mathrm{cm})$, ear height $(\mathrm{cm})$, stem diameter $(\mathrm{cm})$ and ear leaf area $\left(\mathrm{cm}^{2}\right)$.

Statistical analysis: The obtained data were statistically analyzed for anlaysis of variance by using computer statistical program MSTAT-C.The Ten single crosses comprise a half diallel among 5 inbred parents. Data of all 10 single crosses at each nitrogen level treatment were analyzed as randomized blocks. The sum squares of genotypes were partitioned to general and specific combining ability, following method 2 model 1 (fixed) of Griffing (1956).

\section{RESULTS AND DISCUSSION}

Results in table 1 indicated that mean square of genotypes were highly significant for all studied traits under both nitrogen levels i.e., tasselling date, anthesis date, silking date, ASI, plant height, ear height and stem diameter except, ear leaf area under normal nitrogen level. Mean squares for general combining ability (GCA) were significant or highly significant for all studied traits under both nitrogen levels except, ASI under stress nitrogen level, ear leaf area and stem diameter under normal nitrogen level were nonsignificant. Mean quares for specific combining ability (SCA) were highly significant for all studied traits under both nitrogen levels except, ear leaf area under normal nitrogen level. The GCA/SCA ratio was less than unity for ASI, ear height and stem diameter this means that these traits are predominantly controlled by non-additive gene action under both nitrogen levels, also, anthesis date, silking date and plant height under normal nitrogen level as well as ear leaf area under stress nitrogen level show same trend while, anthesis date, silking date and plant height under stress nitrogen level and 
Sultan, M. S. et al.

ear leaf area under normal nitrogen level were controlled by additive gene action, which the GCA/SCA ratio was more than one, as shown in Table 1. Similar results were obtained by El-Hosary et al. (1994), Nawar et al. (2002), Katta et al. (2007) and El-Ghonemy and Ibrahim (2010).

Table 1: Mean squares of genotypes, GCA and SCA for studied maize flowering and vegetative traits under normal and stress nitrogen levels conditions.

\begin{tabular}{|l|c|c|c|c|c|c|c|}
\hline \multicolumn{1}{|c|}{ Traits } & \multicolumn{2}{c|}{ Anthesis date (day) } & \multicolumn{2}{c|}{ Silking date (day) } & \multicolumn{2}{c|}{ ASI (day) } \\
\hline \multicolumn{1}{|c|}{ S.V. } & d.f. & Normal & Stress & Normal & Stress & Normal & Stress \\
\hline Genotypes & 14 & $26.75^{\star *}$ & $24.26^{\star *}$ & $24.21^{\star *}$ & $20.61^{\star *}$ & $0.85^{\star *}$ & $0.59^{\star *}$ \\
\hline GCA & 4 & $5.44^{\star *}$ & $8.89^{\star *}$ & $6.31^{\star *}$ & $9.11^{\star *}$ & $0.28^{*}$ & 0.06 \\
\hline SCA & 10 & $10.31^{\star *}$ & $7.77^{\star *}$ & $8.77^{\star *}$ & $5.98^{\star *}$ & $0.29^{\star *}$ & $0.25^{\star *}$ \\
\hline Error & 28 & 0.44 & 0.69 & 0.44 & 0.68 & 0.08 & 0.06 \\
\hline GCA/SCA & & 0.53 & 1.14 & 0.72 & 1.52 & 0.97 & 0.24 \\
\hline
\end{tabular}

Table 1: Continue

\begin{tabular}{|c|c|c|c|c|c|}
\hline Traits & $\begin{array}{c}\text { Plant height } \\
(\mathbf{c m})\end{array}$ & Ear height $\mathbf{( c m )}$ & $\begin{array}{c}\text { Ear leaf area } \\
\left(\mathrm{cm}^{2}\right)\end{array}$ & $\begin{array}{c}\text { Stem } \\
\text { diameter }(\mathrm{cm})\end{array}$ \\
\hline
\end{tabular}

S.V. d.f. Normal Stress Normal Stress Normal Stress Normal Stress Genotypes $145562.1^{\star \star} 5446.7^{\star \star} 1725.1^{\star \star} 1748.7^{\star \star} 794133.577908 .0^{\star \star} 0.38^{\star \star} \quad 0.36^{\star \star}$ \begin{tabular}{|l|l|l|l|l|l|l|l|l}
\hline GCA & 4 & $1680.2^{* *}$ & $1969.8^{\star \star}$ & $317.2^{\star *}$ & $422.1^{* \star}$ & $576528.012516 .6^{* \star}$ & 0.03 & $0.02^{\star *}$ \\
\hline
\end{tabular}

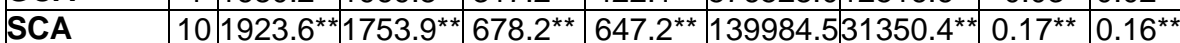

\begin{tabular}{|l|c|c|c|c|c|c|c|c|c|}
\hline Error & 28 & 16.40 & 34.60 & 9.815 & 14.42 & 276049.2 & 351.5 & 0.01 & 0.0001 \\
\hline
\end{tabular}
\begin{tabular}{|l|l|l|l|l|l|l|l|l|}
\hline GCA/SCA & 0.87 & 1.12 & 0.47 & 0.65 & 4.12 & 0.40 & 0.18 & 0.13 \\
\hline
\end{tabular} ${ }^{*}$ and $^{\star \star}$ significant at $5 \%$ and $1 \%$ probability levels, respectively.

Table 2: Means of studied traits for maize inbreds and their crosses under normal and stress nitrogen levels.

\begin{tabular}{|c|c|c|c|c|c|c|}
\hline Traits & \multicolumn{2}{|c|}{ Anthesis date (day) } & \multicolumn{2}{|c|}{ Silking date (day) } & \multicolumn{2}{|c|}{ ASI (day) } \\
\hline Genotypes & Stress & Normal & Stress & Normal & Stress & Normal \\
\hline$P_{1}($ Inb.84) & 63.00 & 68.67 & 64.00 & 70.33 & 1.00 & 1.67 \\
\hline $\mathrm{P}_{2}(\operatorname{Inb} .95)$ & 62.00 & 68.00 & 62.67 & 69.33 & 0.67 & 1.33 \\
\hline $\mathrm{P}_{3}(\operatorname{Inb} .144)$ & 63.33 & 68.67 & 64.33 & 70.67 & 1.00 & 2.00 \\
\hline$P_{4}(\operatorname{Inb} .204)$ & 65.33 & 69.00 & 66.33 & 71.00 & 1.00 & 2.00 \\
\hline$P_{5}(\operatorname{Inb} .213)$ & 62.33 & 66.67 & 63.00 & 68.33 & 0.67 & 1.67 \\
\hline Parents mean & 63.20 & 68.20 & 12.60 & 13.67 & 0.87 & 1.73 \\
\hline$P_{1} \times P_{2}$ & 59.67 & 63.67 & 60.67 & 65.33 & 1.00 & 1.67 \\
\hline$P_{1} \times P_{3}$ & 59.00 & 64.67 & 60.00 & 66.33 & 1.00 & 1.67 \\
\hline $\mathbf{P}_{1} \times P_{4}$ & 63.67 & 68.33 & 65.33 & 70.67 & 1.67 & 2.33 \\
\hline$P_{1} \times P_{5}$ & 60.00 & 65.00 & 61.33 & 67.33 & 1.33 & 2.33 \\
\hline $\mathrm{P}_{2} \times \mathrm{P}_{3}$ & 55.33 & 60.33 & 57.00 & 62.67 & 1.67 & 2.33 \\
\hline $\mathbf{P}_{2} \times \mathbf{P}_{4}$ & 61.33 & 66.00 & 62.67 & 68.67 & 1.33 & 2.67 \\
\hline $\mathbf{P}_{2} \times \mathbf{P}_{5}$ & 55.33 & 60.00 & 57.33 & 63.00 & 2.00 & 3.00 \\
\hline $\mathbf{P}_{3} \times P_{4}$ & 59.00 & 62.67 & 60.67 & 65.33 & 1.67 & 2.67 \\
\hline$P_{3} \times P_{5}$ & 59.67 & 68.00 & 61.67 & 70.67 & 2.00 & 2.67 \\
\hline$P_{4} \times P_{5}$ & 60.67 & 67.00 & 62.33 & 70.00 & 1.67 & 3.00 \\
\hline Check S.C.10 & 64.33 & 71.33 & 65.67 & 73.67 & 1.33 & 2.33 \\
\hline Crosses mean & 59.82 & 65.18 & 61.33 & 67.61 & 1.52 & 2.42 \\
\hline L.S.D. at 0.05 & 2.40 & 1.93 & 2.39 & 1.93 & 0.71 & 0.82 \\
\hline L.S.D. at 0.01 & 3.24 & 2.60 & 3.22 & 2.60 & 0.95 & 1.11 \\
\hline
\end{tabular}


Table 2: Continue...

\begin{tabular}{|c|c|c|c|c|c|c|c|c|}
\hline \multirow{2}{*}{\begin{tabular}{|c|} 
Traits \\
Genotypes \\
\end{tabular}} & \multicolumn{2}{|c|}{ Plant height(cm) } & \multicolumn{2}{|c|}{ Ear height(cm) } & \multicolumn{2}{|c|}{$\begin{array}{c}\text { Ear leaf } \\
\text { area }\left(\mathrm{cm}^{2}\right)\end{array}$} & \multicolumn{2}{|c|}{$\begin{array}{c}\text { Stem } \\
\text { diameter(cm) }\end{array}$} \\
\hline & Stress & Normal & Stress & Normal & Stress & Normal & Stress & Normal \\
\hline$P_{1}(\operatorname{Inb} .84)$ & 204.0 & 217.6 & 126.9 & 136.8 & 381.7 & 501.6 & 2.73 & 2.95 \\
\hline $\mathrm{P}_{2}(\operatorname{Inb} .95)$ & 162.8 & 181.1 & 104.6 & 120.7 & 524.6 & 649.6 & 2.58 & 3.03 \\
\hline $\mathrm{P}_{3}(\operatorname{Inb} .144)$ & 180.9 & 197.9 & 110.6 & 124.1 & 490.3 & 624.8 & 2.60 & 2.82 \\
\hline$P_{4}(\operatorname{Inb} .204)$ & 216.8 & 232.8 & \begin{tabular}{|l|}
113.7 \\
\end{tabular} & 125.9 & 582.9 & 688.2 & 2.75 & 2.95 \\
\hline$P_{5}(\operatorname{lnb} .213)$ & 140.0 & 159.9 & 97.1 & 113.2 & 494.8 & 646.8 & 2.15 & 2.35 \\
\hline $\begin{array}{l}\text { Parents } \\
\text { mean }\end{array}$ & 180.9 & 197.9 & 110.6 & 124.1 & 494.9 & 622.2 & 2.56 & 2.82 \\
\hline $\mathrm{P}_{1} \times \mathrm{P}_{2}$ & 260.0 & 279.7 & 159.2 & 177.2 & 675.7 & 797.9 & 3.07 & 2.90 \\
\hline$P_{1} \times P_{3}$ & 266.3 & 284.8 & 160.4 & 175.2 & 797.2 & 921.4 & 3.08 & 3.33 \\
\hline$P_{1} \times P_{4}$ & 293.1 & 307.9 & \begin{tabular}{|l|}
178.2 \\
\end{tabular} & 188.6 & 765.5 & 879.1 & 3.15 & 3.43 \\
\hline$P_{1} X P_{5}$ & 237.9 & 256.7 & 152.2 & 166.9 & 733.4 & 876.5 & 3.25 & 3.45 \\
\hline $\mathrm{P}_{2} \times \mathrm{P}_{3}$ & 235.7 & 261.7 & 148.6 & 167.0 & 810.8 & 969.2 & 3.20 & 3.44 \\
\hline $\mathrm{P}_{2} \times \mathrm{P}_{4}$ & 260.0 & 285.1 & 155.6 & 174.4 & 839.1 & 973.9 & 3.23 & 3.47 \\
\hline$P_{2} \times P_{5}$ & 212.7 & 237.4 & 137.3 & 151.4 & 746.0 & 847.5 & 3.23 & 3.47 \\
\hline $\mathrm{P}_{3} \times \mathrm{P}_{4}$ & 270.0 & 289.1 & 164.7 & 172.3 & 929.9 & 1072.7 & 3.23 & 3.52 \\
\hline $\mathrm{P}_{3} \times \mathrm{P}_{5}$ & 242.6 & 260.7 & 139.9 & 151.6 & 816.6 & 992.9 & 3.24 & 3.48 \\
\hline$P_{4} \times P_{5}$ & 247.6 & 270.4 & 135.5 & 157.3 & 828.6 & 956.0 & 3.35 & 3.59 \\
\hline $\begin{array}{l}\text { Check } \\
\text { S.C.10 } \\
\end{array}$ & 257.1 & 284.5 & 128.1 & 154.8 & 8921.0 & 1079.9 & 3.22 & 3.79 \\
\hline $\begin{array}{l}\text { Crosses } \\
\text { mean }\end{array}$ & 253.0 & 274.4 & 150.9 & 167.0 & 803.2 & 942.8 & 3.20 & 3.44 \\
\hline $\begin{array}{ll}\text { L.S.D. } & \text { at } \\
0.05 & \end{array}$ & 17.04 & 11.73 & 11.00 & 9.07 & 54.30 & - & 0.18 & 0.31 \\
\hline 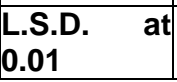 & 22.98 & 15.82 & 14.84 & 12.24 & 73.25 & - & 0.24 & 0.42 \\
\hline
\end{tabular}

\section{Mean performance of traits:}

Means of number of days to $50 \%$ anthesis as affected by normal and stress nitrogen levels are presented in Table (2). The earliest parent in pollen shedding was $\mathrm{P}_{2}$ (Inb.95) under stress nitrogen level and $\mathrm{p}_{5}$ (Inb.213) under normal nitrogen levels. The earliest crosses in pollen shedding were $p_{2} \times p_{5}$ under normal nitrogen level and $\mathrm{P}_{2} \mathrm{P}_{3}$ under stress nitrogen level. The earliest parents in silking date was $P_{5}$ (Inb.213) under normal nitrogen level and $\mathrm{P}_{2}$ (Inb.95) under stress nitrogen level. The earliest crosses in silking date were $\mathrm{p}_{2} \times \mathrm{p}_{3}$ under normal nitrogen level and $\mathrm{P}_{2} \times \mathrm{P}_{3}$ under stress nitrogen level. The earliest parent in (ASI) was $P_{2}(\operatorname{Inb} .95)$ and $P_{5}(\operatorname{Inb} .213)$ under stress nitrogen level and $\mathrm{P}_{2}(\mathrm{Inb} .95)$ under normal nitrogen level. The earliest crosses in ASI were $\mathrm{P}_{1} \times \mathrm{P}_{2}$ and $\mathrm{P}_{1} \times \mathrm{P}_{3}$ under normal nitrogen level and $\mathrm{P}_{1} \times \mathrm{P}_{2}$ and $\mathrm{P}_{1} \times \mathrm{P}_{3}$ under stress nitrogen level. The tallest parent was $\mathrm{P}_{4}$ (Inb.204) under both nitrogen levels. Mean while, parent $\mathrm{P}_{5}(\operatorname{Inb} .213)$ was the shortest parent under both nitrogen levels. The tallest cross was $\mathrm{P}_{1} \times \mathrm{P}_{4}$ under both nitrogen levels. Meanwhile, cross $\mathrm{P}_{2} \times \mathrm{P}_{5}$ under both nitrogen levels was the shortest crosses. The highest parent in ear height was $\mathrm{P}_{1}$ (Inb.84) under both nitrogen levels. The highest cross in ear height 
was $\mathrm{P}_{1} \times \mathrm{P}_{4}$ under both nitrogen levels. Meanwhile, crosses $\mathrm{P}_{2} \mathrm{XP}_{5}$ under normal nitrogen level and S.C.10 under stress nitrogen level were the lowest crosses. It may indicated that ear height is greatly influenced by different agronomic treatments. These results are in agreement with findings by Abdel-Monaem, (2000) and Oliverira (2011). Ear leaf area ranged from 381.7 to $582.9 \mathrm{~cm}^{2}$ under stress nitrogen level. The highest values were recorded by $\mathrm{P}_{4}(\mathrm{Inb} .204)$ under both nitrogen levels. . Ear leaf area ranged from 675.7 to $929.9 \mathrm{~cm}^{2}$ under stress nitrogen level. The highest value was recorded by $\mathrm{P}_{3} \mathrm{XP}_{4}$ under stress nitrogen level. Stem diameter ranged from 2.35 to $3.03 \mathrm{~cm}$ under normal nitrogen level. While, under stress nitrogen level ranged from 2.15 to $2.75 \mathrm{~cm}$. The highest value 3.03 $\mathrm{cm}$ was recorded by $\mathrm{P}_{2}(\mathrm{Inb} .95)$ under normal nitrogen level and $\mathrm{P}_{4}(\mathrm{Inb} .204) 2.75 \mathrm{~cm}$ under stress nitrogen level. Stem diameter ranged from 2.9 to $3.79 \mathrm{~cm}$ under normal nitrogen level, and from 3.07 to $3.35 \mathrm{~cm}$ under stress nitrogen level. The highest value was recorded by S.C.10 under normal nitrogen level and $\mathrm{P}_{4} \mathrm{XP}_{5}$ under stress nitrogen level.

General combining ability: Based on GCA estimates, it could be concluded that the best combiners for anthesis date and silking date was inbred line $\mathrm{P}_{2}$ (Inb.95), for plant height, ear height was $\mathrm{P}_{5}(\operatorname{Inb} .213)$ and ear leaf area was $\mathrm{P}_{4}(\operatorname{Inb} .204)$. These results indicated that these inbred lines could be as good combiners for improving these traits (Table,2 ).

Table 3: Estimates of G.C.A. effects of five parents maize genotypes:

\begin{tabular}{|l|c|c|c|c|c|c|}
\hline \multicolumn{1}{|c|}{ Traits } & \multicolumn{2}{|c|}{ Anthesis date (day) } & \multicolumn{2}{c|}{ Silking date (day) } & \multicolumn{2}{c|}{ ASI (day) } \\
\hline Parent & Stress & Normal & Stress & Normal & Stress & Normal \\
\hline $\mathbf{P}_{\mathbf{1}}$ (Inb.84) & 0.64 & 0.62 & 0.51 & 0.35 & -0.12 & -0.27 \\
\hline $\mathbf{P}_{\mathbf{2}}$ (Inb.95) & $-1.17^{\star}$ & $-1.24^{\star *}$ & $-1.25^{\star}$ & $-1.36^{\star *}$ & -0.08 & -0.12 \\
\hline $\mathbf{P}_{\mathbf{3}}$ (Inb.144) & -0.60 & -0.24 & -0.53 & -0.22 & 0.07 & 0.02 \\
\hline $\mathbf{P}_{\mathbf{4}}$ (Inb. 204) & $1.64^{\star *}$ & $1.05^{\star}$ & $1.70^{* \star}$ & $1.26^{\star *}$ & 0.07 & 0.21 \\
\hline $\mathbf{P}_{\mathbf{5}}$ (Inb. 213) & -0.50 & -0.19 & -0.44 & -0.03 & 0.07 & 0.16 \\
\hline S. E. (gi)1 & $\mathbf{0 . 2 8}$ & $\mathbf{0 . 2 3}$ & $\mathbf{0 . 2 8}$ & $\mathbf{0 . 2 2}$ & $\mathbf{0 . 0 8}$ & $\mathbf{0 . 1 0}$ \\
\hline S. E. (gi - gj)2 & $\mathbf{0 . 4 4}$ & $\mathbf{0 . 3 6}$ & $\mathbf{0 . 4 4}$ & $\mathbf{0 . 3 6}$ & $\mathbf{0 . 1 3}$ & $\mathbf{0 . 1 5}$ \\
\hline
\end{tabular}

Table 3: Continue.

\begin{tabular}{|c|c|c|c|c|c|c|c|c|}
\hline \multirow{2}{*}{$\begin{array}{l}\text { Traits } \\
\text { Parent }\end{array}$} & \multicolumn{2}{|c|}{ Plant height (cm) } & \multicolumn{2}{|c|}{ Ear height (cm) } & \multicolumn{2}{|c|}{ Ear leaf area $\left(\mathrm{cm}^{2}\right)$} & \multicolumn{2}{|c|}{$\begin{array}{l}\text { Stem diameter } \\
(\mathbf{c m})\end{array}$} \\
\hline & Stress & Normal & Stress & Nor & Stress & Normal & Stress & Normal \\
\hline$P_{1}(\operatorname{Inb} .84)$ & $13.30^{*}$ & $10.73^{\star *}$ & $9.99^{\star *}$ & $63^{\star *}$ & $-61.65^{\star *}$ & -185.44 & 0.01 & -0.04 \\
\hline$P_{2}(\operatorname{lnb} .95)$ & $-11.17^{* *}$ & $-9.00^{* *}$ & -3.41 & -1.36 & -6.55 & -126.94 & -0.01 & 01 \\
\hline$P_{3}(\operatorname{lnb} .144)$ & 0.61 & 0.41 & 0.14 & -0.96 & $24.03^{*}$ & -81.49 & 0.00 & 0.02 \\
\hline$P_{4}$ (Inb. 204) & $18.88^{\star \star}$ & $18.44^{\star \star}$ & $3.95^{\star}$ & $3.34^{*}$ & $51.71^{\star *}$ & $508.97^{\star}$ & $0.07^{*}$ & 0.09 \\
\hline$P_{5}(\operatorname{lnb} .213)$ & $-21.61^{* *}$ & \begin{tabular}{|l}
$-20.59^{\star *}$ \\
\end{tabular} & $-10.68^{\star *}$ & $-9.64^{\star *}$ & -7.53 & -115.10 & $-0.08^{*}$ & -0.08 \\
\hline S. E. $\left(g_{i}\right)$ & 1.99 & 1.37 & 1.28 & 1.06 & 6.34 & 177.62 & 0.02 & 0.04 \\
\hline S. E. $\left(g_{i}-g_{j}\right)$ & 3.14 & 2.16 & 2.03 & 1.67 & 10.02 & 280.84 & 0.03 & 0.06 \\
\hline
\end{tabular}

*, ** significant at 0.05 and 0.01 level of probability , respectively

S.E.(gi), standard error for an GCA effects.

S.E.(gi-gj), standard error for the difference between two estimates of GCA effects.

Specific combining ability effects $\left(S_{i j}\right)$ : Significant SCA effects were found in all studied traits for most crosses under both nitrogen levels (Table,3). Based on SCA effects, it could be concluded that under both nitrogen levels 
highly significant negative SCA effects for anthesis date and silking date were recorded with $\mathrm{P}_{2}$ (Inb.95) X $\mathrm{P}_{3}$ (Inb.144) and $\mathrm{P}_{3}$ (Inb.144) X $\mathrm{P}_{4}$ (Inb.204) while, the positive significant for ear height was $\mathrm{P}_{1}(\operatorname{Inb} .84) \mathrm{XP}_{4}(\operatorname{Inb} .204)$, for plant height was $P_{3}$ (Inb.144) $X P_{5}$ (Inb.213), for stem diameter was $\mathrm{P}_{4}(\operatorname{Inb} .204) X \mathrm{P}_{5}(\operatorname{Inb} .213)$ under both nitrogen levels for ear leaf area was $P_{3}($ Inb.144) XP 4 (Inb.204) under stress nitrogen level. These crosses could be selected and used in hybridization programs for improving these traits.

Table (4): Estimates of S.C.A. effects of 10 single crosses maize for flowering and vegetative traits under normal and stress nitrogen levels.

\begin{tabular}{|c|c|c|c|c|c|c|}
\hline Traits & \multicolumn{2}{|c|}{ Anthesis date (day) } & \multicolumn{2}{|c|}{ Silking date (day) } & \multicolumn{2}{|c|}{ A.S.I. (day) } \\
\hline Crosses & Stress & Normal & Stress & Normal & Stress & Normal \\
\hline$P_{1} \times P_{2}$ & -0.44 & $-1.49^{*}$ & -0.56 & $-1.64^{*}$ & -0.11 & -0.14 \\
\hline$P_{1} X P_{3}$ & $-1.68^{*}$ & $-1.49^{*}$ & $-1.94^{*}$ & $-1.78^{*}$ & -0.25 & -0.29 \\
\hline$P_{1} X P_{4}$ & 0.75 & 0.89 & 1.16 & 1.08 & 0.41 & 0.19 \\
\hline$P_{1} \times P_{5}$ & -0.78 & -1.21 & -0.70 & -0.97 & 0.08 & 0.24 \\
\hline$P_{2} \times P_{3}$ & $-3.54^{\star \star}$ & $-3.97^{\star \star}$ & $-3.18^{\star \star}$ & $-3.73^{\star \star}$ & 0.37 & 0.24 \\
\hline $\mathbf{P}_{2} \times \mathbf{P}_{4}$ & 0.22 & 0.41 & 0.25 & 0.79 & 0.03 & 0.38 \\
\hline$P_{2} \times P_{5}$ & $-3.64^{\star *}$ & $-4.35^{\star *}$ & $-2.94^{* *}$ & $-3.59^{\star *}$ & $0.70^{* *}$ & $0.76^{*}$ \\
\hline $\mathbf{P}_{3} X P_{4}$ & $-2.68^{* *}$ & $-3.92^{* *}$ & $-2.46^{\star *}$ & $-3.68^{* *}$ & 0.22 & 0.24 \\
\hline$P_{3} \times P_{5}$ & 0.13 & $2.65^{\star \star}$ & 0.68 & $2.94^{* \star}$ & $0.56^{*}$ & 0.29 \\
\hline$P_{4} \times P_{5}$ & -1.11 & 0.37 & -0.89 & 0.79 & 0.22 & 0.43 \\
\hline S. E. $\left(S_{i j}\right)$ & 0.72 & 0.58 & 0.72 & 0.58 & 0.21 & 0.25 \\
\hline S. E. $\left(S_{i j}-S_{i k}\right)$ & 1.09 & 0.87 & 1.08 & 0.87 & 0.32 & 0.37 \\
\hline S. E. $\left(\mathrm{S}_{\mathrm{ij}}-\mathrm{S}_{\mathrm{kl}}\right)$ & 0.99 & 0.80 & 0.99 & 0.79 & 0.29 & 0.34 \\
\hline
\end{tabular}

Table 4: Continue.

\begin{tabular}{|c|c|c|c|c|c|c|c|c|}
\hline \multirow{2}{*}{\begin{tabular}{|c|} 
Traits \\
Crosses \\
\end{tabular}} & \multicolumn{2}{|c|}{ Plant height $(\mathrm{cm})$} & \multicolumn{2}{|c|}{ Ear height (cm) } & \multicolumn{2}{|c|}{ Ear leaf area $\left(\mathrm{cm}^{2}\right)$} & \multicolumn{2}{|c|}{$\begin{array}{c}\text { Stem diameter } \\
\text { (cm) }\end{array}$} \\
\hline & Stress & Normal & Stress & Normal & Stress & Normal & Stress & Normal \\
\hline $\mathbf{P}_{1} \mathbf{X} \mathbf{P}_{2}$ & $29.18^{* *}$ & $29.75^{\star \star}$ & $13.63^{* *}$ & $16.47^{* *}$ & $49.45^{\star}$ & 147.50 & 0.07 & $-0.28^{*}$ \\
\hline $\mathbf{P}_{1} \mathbf{X} \mathbf{P}_{3}$ & $23.73^{\star *}$ & $25.48^{\star \star}$ & $11.28^{\star \star}$ & $14.04^{\star \star}$ & $140.37^{\star *}$ & .60 & 0.08 & 0.14 \\
\hline $\mathbf{P}_{1} \mathbf{X P} \mathbf{P}_{4}$ & 32. & & & & & & 0.07 & 17 \\
\hline$\overline{\mathbf{P}_{1} \mathbf{X} \mathbf{P}_{5}}$ & $17.55^{\star \star}$ & $18.38^{\star *}$ & $13.93^{\star *}$ & $14.37^{* *}$ & $108.13^{\star *}$ & 214.30 & $0.33^{* \star}$ & $0.36^{\star \star}$ \\
\hline $\mathbf{P}_{2} \times P_{3}$ & $17.60^{\star *}$ & $22.10^{* *}$ & $12.95^{\star \star}$ & $15.80^{\star \star}$ & $98.93^{\star *}$ & 215.00 & $0.22^{\star *}$ & 0.20 \\
\hline $\mathbf{P}_{2} \times \mathbf{P}_{4}$ & $23.59^{\star *}$ & $27.50^{\star \star}$ & $16.10^{\star \star}$ & $18.97^{\star \star}$ & $99.42^{\star \star}$ & -370.80 & $0.17^{\star \star}$ & 0.15 \\
\hline $\mathbf{P}_{2} \mathbf{X} \mathbf{P}_{5}$ & $16.82^{\star *}$ & $18.83^{* *}$ & $12.44^{\star *}$ & $8.90^{\star *}$ & $65.65^{\star *}$ & 126.80 & $0.33^{* *}$ & $0.33^{* *}$ \\
\hline$\overline{\mathbf{P}_{3} \mathbf{X P}}$ & 21.8 & & & & & & $0.16^{* \star}$ & 0.19 \\
\hline & & & & & & & ** & $0.34^{* *}$ \\
\hline & $21.67^{\star \star}$ & 24. & & 10. & 89 & 60 & $0.37^{* *}$ & $0.37^{\star \star}$ \\
\hline & 5.1 & & & & 16 & 458.61 & 0.05 & 0.09 \\
\hline $\operatorname{SE}\left(\mathrm{S}_{\mathrm{ij}}-\mathrm{S}_{\mathrm{ik}}\right)$ & 7.70 & 5.30 & 4.97 & 4.10 & 24.55 & 687.92 & 0.08 & 0.14 \\
\hline $\operatorname{SE}\left(\mathrm{S}_{\mathrm{ij}}-\mathrm{S}_{\mathrm{kl}}\right)$ & 7.03 & 4.84 & 4.54 & 3.74 & 22.41 & 627.98 & 0.07 & 0.13 \\
\hline
\end{tabular}

${ }^{*},{ }^{* \star}$ significant at 0.05 and 0.01 level of probability, respectively.

SE(Sij), standard error for an SCA effects.

SE(Sij-Sik), standard error for the difference between two SCA effects for a common parent.

SE(Sij-Skl), standard error for the difference between two SCA effects for a non-common parent.

Heterosis over mid-parents: Results showed significant heterosis for studied traits for all crosses. For anthesis date and silking date were cross $\mathrm{P}_{2} \mathrm{XP}_{3}$ under both nitrogen levels. The highest positive significant for ear 
Sultan, M. S. et al.

height was cross $\mathrm{P} 1 \mathrm{XP} 4$, for plant height was cross $\mathrm{P}_{3} \mathrm{XP}_{5}$ and stem diameter was cross $\mathrm{P}_{4} \mathrm{XP}_{5}$ (Table, 5).

Heterosis over better-parents: Results showed significant heterosis over better-parent in all studied traits for most crosses under both nitrogen levels (Table, 5). For anthesis date and silking date were cross $\mathrm{P}_{2} \mathrm{XP}_{3}$ under both nitrogen levels, for $\mathrm{ASI}$ date was cross $\mathrm{P}_{2} \mathrm{XP}_{5}$ under both nitrogen levels, for ear height was cross $\mathrm{P}_{1} \mathrm{XP}_{4}$ under both nitrogen levels, for plant height was cross $\mathrm{P}_{4} \mathrm{XP}_{5}$ under both nitrogen levels, for ear leaf area was cross $\mathrm{P}_{1} X \mathrm{P}_{3}$ under stress nitrogen level, for stem diameter was cross $\mathrm{P}_{3} \mathrm{XP}_{5}$ under normal nitrogen level, while, cross $\mathrm{P}_{2} \mathrm{XP}_{5}$ under stress nitrogen level.

Table 5: Percentage of heterosis over mid-parent (M.P) and better parent (B.P) for F1 crosses of studied maize flowering and vegetative traits under normal and stress nitrogen levels:

\begin{tabular}{|c|c|c|c|c|c|c|c|c|c|c|c|c|}
\hline Traits & \multicolumn{4}{|c|}{ Anthesis date (day) } & \multicolumn{4}{|c|}{ Silking date (day) } & \multicolumn{4}{|c|}{ ASI (day) } \\
\hline & \multicolumn{2}{|c|}{ Normal } & \multicolumn{2}{|c|}{ Stress } & \multicolumn{2}{|c|}{ Normal } & \multicolumn{2}{|c|}{ Stress } & \multicolumn{2}{|c|}{ Normal } & \multicolumn{2}{|c|}{ Stress } \\
\hline \begin{tabular}{|l|} 
Crosses \\
\end{tabular} & M.P. & B.P. & M.P. & B.P. & M.P. & B.P. & M.P. & B.P. & M.P. & B.P. & M.P. & B.P. \\
\hline$P_{1} \times P_{2}$ & $-6.8^{* \star}$ & $-6.4^{\star *}$ & -4.5 & -3.8 & $-6.4^{\star *}$ & $-5.8^{* *}$ & -4.2 & -3.2 & 11.3 & 25.6 & 19.8 & 49.3 \\
\hline $\mathrm{P}_{1} \times \mathrm{P}_{3}$ & $-5.8^{\star *}$ & $-5.8^{\star \star}$ & $-6.6^{* *}$ & $-6.3^{* \star}$ & $-5.9^{\star \star}$ & $-5.7^{\star \star *}$ & $-6.5^{\star \star}$ & $-6.3^{\star \star}$ & -9.0 & 0.0 & 0.0 & 0.0 \\
\hline $\mathbf{P}_{1} \times P_{4}$ & -0.7 & -0.5 & -0.8 & 1.1 & 0.0 & 0.5 & 0.3 & 2.1 & 27.0 & 39.5 & 67.0 & 7.0 \\
\hline $\mathrm{P}_{1} \times \mathrm{P}_{5}$ & $-3.9^{*}$ & -2.5 & -4.3 & -3.7 & -2.9 & -1.5 & -3.4 & -2.7 & 39.5 & 39.5 & 59.3 & 98.5 \\
\hline $\mathbf{P}_{2} \times P_{3}$ & $-11.7^{* \star}$ & $-11.3^{\star \star}$ & $-11.7^{* \star}$ & $-10.8^{*}$ & $-10.5^{\star \star}$ & $-9.6^{\star *}$ & $-10.2^{\star \star}$ & $-9.0^{\star \star}$ & 39.9 & $75.2^{*}$ & 100.0 & $149.3^{\star \star}$ \\
\hline $\mathrm{P}_{2} \times \mathrm{P}_{4}$ & $-3.6^{*}$ & $-2.9^{*}$ & -3.7 & -1.1 & -2.1 & -1.0 & -2.8 & 0 & $60.4^{*}$ & $100.8^{\star \star}$ & 59.3 & 98.5 \\
\hline $\mathrm{P}_{2} \times \mathrm{P}_{5}$ & $-10.9^{\star \star}$ & $-10.0^{\star \star}$ & $-11.0^{* \star}$ & $-10.8^{\star *}$ & $-8.5^{\star \star}$ & $-7.8^{\star \star}$ & $-8.8^{\star *}$ & $-8.5^{\star \star}$ & $100^{\star \star}$ & $125.6^{\star \star}$ & $198.5^{\star \star}$ & $198.5^{\star \star}$ \\
\hline $\mathrm{P}_{3} \times \mathrm{P}_{4}$ & $-9.0^{* *}$. & $-8.7^{* *}$ & $-8.3^{* *}$ & $-6.8^{* *}$ & $-7.8^{* \star}$ & $-7.6^{* *}$ & $-7.1^{* *}$ & $-5.7^{\star \star}$ & 33.5 & 33.5 & 67.0 & 67.0 \\
\hline $\mathbf{P}_{3} \times P_{5}$ & 0.5 & 2.0 & $-5.0^{*}$ & $-4.3^{*}$ & 1.7 & $3.4^{*}$ & -3.1 & -2.1 & 45.5 & $59.9^{*}$ & $139.5^{\star}$ & $198.5^{\star \star}$ \\
\hline $\mathrm{P}_{4} \times \mathrm{PP}_{5}$ & -1.2 & 0.5 & $-5.0^{\star}$ & -2.7 & 0.5 & 2.4 & -3.6 & -1.1 & $63.5^{*}$ & $79.6^{* *}$ & 100 & $149.3^{* \star}$ \\
\hline $\begin{array}{l}\text { L.S.D. at } \\
5 \%\end{array}$ & 2.4 & 1.9 & 2.9 & 2.4 & 2.4 & 1.9 & 2.9 & 2.4 & 1.0 & 0.8 & 0.9 & 0.7 \\
\hline $\begin{array}{l}\text { L.S.D. at } \\
1 \%\end{array}$ & 3.2 & 2.6 & 4.0 & 3.2 & 3.2 & 2.6 & 3.9 & 3.2 & 1.4 & 1.1 & 1.2 & 1.0 \\
\hline
\end{tabular}

Table 5: Continue.

\begin{tabular}{|c|c|c|c|c|c|c|c|c|}
\hline Traits & \multicolumn{4}{|c|}{ Ear height (cm) } & \multicolumn{4}{|c|}{ Plant height (cm) } \\
\hline & \multicolumn{2}{|c|}{ Normal } & \multicolumn{2}{|c|}{ Stress } & \multicolumn{2}{|c|}{ Normal } & \multicolumn{2}{|c|}{ Stress } \\
\hline Crosses & M.P. & B.P. & M.P. & B.P. & M.P. & B.P. & M.P. & B.P. \\
\hline $\mathbf{P}_{1} \times P_{2}$ & $37.7^{\star \star}$ & $46.8^{\star \star}$ & $37.5^{\star \star}$ & $52.2^{\star *}$ & $40.3^{* *}$ & $54.4^{\star \star}$ & $41.8^{\star *}$ & $59.7^{* \star}$ \\
\hline$P_{1} \times P_{3}$ & $34.3^{\star \star}$ & $41.2^{\star \star}$ & $35.1^{\star \star}$ & $45.0^{\star \star}$ & $37.1^{\star \star}$ & $43.9^{\star \star}$ & $38.4^{\star *}$ & $47.2^{* *}$ \\
\hline $\mathbf{P}_{1} \times P_{4}$ & $43.6^{\star \star}$ & $49.8^{\star \star}$ & $48.1^{\star \star}$ & $56.7^{\star \star}$ & $36.7^{\star \star}$ & $41.5^{\star \star}$ & $39.3^{\star *}$ & $43.7^{* \star}$ \\
\hline $\mathbf{P}_{1} \times P_{5}$ & $33.5^{\star \star}$ & $47.5^{\star *}$ & $35.9^{* *}$ & $56.8^{\star *}$ & $36.0^{* *}$ & $60.5^{\star \star}$ & $38.3^{\star *}$ & $70.0^{* *}$ \\
\hline $\mathbf{P}_{2} \times P_{3}$ & $36.4^{\star \star}$ & $38.3^{* *}$ & $38.2^{* \star}$ & $42.1^{\star *}$ & $38.1^{* *}$ & $44.5^{\star *}$ & $37.2^{\star *}$ & $44.8^{* *}$ \\
\hline $\mathbf{P}_{2} \times P_{4}$ & $41.5^{\star *}$ & $44.5^{* *}$ & $42.6^{\star \star}$ & $48.8^{* *}$ & $37.8^{* *}$ & $57.4^{\star *}$ & $37.0^{* *}$ & $59.7^{* \star}$ \\
\hline $\mathbf{P}_{2} \times P_{5}$ & $29.5^{\star \star}$ & $33.8^{\star \star}$ & $36.2^{\star \star}$ & $41.4^{\star \star}$ & $39.3^{* \star}$ & $48.5^{\star \star}$ & $40.5^{\star \star}$ & $52.0^{* *}$ \\
\hline $\mathbf{P}_{3} \times P_{4}$ & $37.9^{\star *}$ & $38.9^{* *}$ & $46.9^{\star \star}$ & $49.0^{\star *}$ & $34.2^{\star \star}$ & $46.1^{\star *}$ & $35.8^{* *}$ & $49.3^{\star *}$ \\
\hline $\mathbf{P}_{3} \times P_{5}$ & $27.8^{\star \star}$ & $34.0^{\star \star}$ & 34.7 & $44.1^{\star \star}$ & $45.7^{\star \star}$ & $63.0^{* *}$ & $51.2^{\star \star}$ & $73.3^{* *}$ \\
\hline $\mathbf{P}_{4} \times P_{5}$ & $31.6^{\star \star}$ & $39.0^{* *}$ & $28.5^{\star \star}$ & $39.6^{* *}$ & $37.7^{\star \star}$ & $69.1^{\star \star}$ & $38.8^{* *}$ & $76.9^{* *}$ \\
\hline L.S.D.at 5\% & 11.1 & 9.1 & 13.5 & 11.0 & 14.4 & 11.7 & 20.9 & 17.1 \\
\hline L.S.D.at 1\% & 15.0 & 12.2 & 18.2 & 14.8 & 19.4 & 15.8 & 28.1 & 23.0 \\
\hline
\end{tabular}


Table(5) Continue.

\begin{tabular}{|c|c|c|c|c|c|c|c|c|}
\hline \multirow{3}{*}{$\begin{array}{c}\text { Traits } \\
\text { Crosses }\end{array}$} & \multicolumn{4}{|c|}{ Stem diameter (cm) } & \multicolumn{4}{|c|}{ Ear leaf area $\left(\mathrm{cm}^{2}\right)$} \\
\hline & \multicolumn{2}{|c|}{ Normal } & \multicolumn{2}{|c|}{ Stress } & \multicolumn{2}{|c|}{ Normal } & \multicolumn{2}{|c|}{ Stress } \\
\hline & M.P. & B.P. & M.P. & B.P. & M.P. & B.P. & M.P. & B.P. \\
\hline $\mathbf{P}_{1} \times P_{2}$ & -3.0 & -4.3 & $15.6^{\star \star}$ & $12.5^{\star \star}$ & 38.6 & 22.8 & $49.1^{\star \star}$ & $28.8^{\star \star}$ \\
\hline $\mathbf{P}_{1} \times P_{3}$ & $15.4^{*}$ & $12.9^{*}$ & $15.6^{\star *}$ & $12.8^{* *}$ & 63.6 & 47.5 & $82.9^{* *}$ & $62.6^{\star *}$ \\
\hline $\mathbf{P}_{1} \times P_{4}$ & $16.3^{\star \star}$ & $16.3^{\star *}$ & $15.0^{\star \star}$ & $14.5^{\star \star}$ & 47.8 & 36.0 & $58.7^{\star \star}$ & $31.3^{* \star}$ \\
\hline $\mathbf{P}_{1} \times P_{5}$ & $30.2^{\star \star}$ & $16.9^{\star \star}$ & $33.2^{\star \star}$ & $19.0^{\star *}$ & 52.6 & 35.5 & $67.4^{\star \star}$ & 48.2 \\
\hline $\mathbf{P}_{2} \times P_{3}$ & $17.6^{\star \star}$ & $13.5^{\star *}$ & $23.6^{\star \star}$ & $23.1^{\star *}$ & 52.1 & 49.2 & $59.8^{* *}$ & $54.6^{* *}$ \\
\hline $\mathbf{P}_{2} \times P_{4}$ & $16.1^{* \star}$ & $14.5^{\star *}$ & $21.2^{\star \star}$ & $17.5^{\star \star}$ & 45.6 & 41.5 & $51.5^{\star *}$ & $43.9^{* *}$ \\
\hline $\mathbf{P}_{2} \times P_{5}$ & $29.0^{* *}$ & $14.5^{\star \star}$ & $36.6^{\star *}$ & $25.2^{\star *}$ & 30.7 & 30.5 & $46.4^{* *}$ & $42.2^{\star *}$ \\
\hline $\mathbf{P}_{3} \times \mathbf{P}_{4}$ & $22.0^{* *}$ & $19.3^{\star *}$ & $20.7^{\star *}$ & $17.5^{\star *}$ & 63.4 & 55.9 & $73.3^{* *}$ & $59.5^{\star *}$ \\
\hline $\mathbf{P}_{3} \times P_{5}$ & $34.6^{\star *}$ & $23.4^{\star *}$ & $36.4^{\star *}$ & $24.6^{\star *}$ & 56.2 & 53.5 & $65.8^{* *}$ & $65.0^{\star \star}$ \\
\hline $\mathbf{P}_{4} \times P_{5}$ & $35.5^{\star \star}$ & $21.7^{* \star}$ & $36.7^{\star \star}$ & $21.8^{\star \star}$ & 43.8 & 39.5 & $53.8^{\star \star}$ & $42.1^{* \star}$ \\
\hline L.S.D. at 5\% & 04 & 0.3 & 0.2 & 0.2 & 1865.6 & 1523.2 & 66.6 & 54.4 \\
\hline L.S.D. at $1 \%$ & 0.5 & 0.4 & 0.3 & 0.2 & 2511.7 & 2050.8 & 89.6 & 73.2 \\
\hline
\end{tabular}

${ }^{*},{ }^{* *}$ significant at 0.05 and 0.01 level of probability, respectively.

\section{REFERENCES}

Abdel-Monaem, M. A. (2000). Breeding studies on maize tolerance to low nitrogen fertilization. M.Sc. Thesis Agric. Sci. (Agronomy), Faculty of Agric. Mansoura Univ.Egypt.

Ahmed, M.A.; M.H. El-Sheikh and Sh.A. EL-Shamarka (2000). Diallel analysis of yielding ability and earliness in maize. J. Agric. Sci. Mansoura Univ., 25 (7): 3717-3726.

Akintoye, H. A; J. G. Kling, and E. O. Lucase (1999). N-use efficiency of single, double and synthetic maize lines grown at four $\mathrm{N}$ levels in three ecological zones of West Africa. Field Crops Res., $60: 3,189-199$.

Alamnie, A., M.C. Wali, P.M. Salimath and R.C.Jagadeesha (2006). Combining ability and heterosis for grain yield and ear characters in maize. Karnata j. Agric. Sci., 19:13-16.

Al-Naggar, A. M., M.S. Radwan and M.M.M. Atta (2002). Analysis of diallel crosses among ten maize populations differing in drought tolerance. Egypt .J. Plant Breed, 6:179-198.

Barakat, A.A. and M.M.A. Osman (2008). Evaluation of some newly developed yellow maize inbred lines for combining ability in two location. J.Agric.Sci .Mansoura Univ., 33(7): 4667-4679.

Dadheech, A. and V.N. Joshi (2007). Heterosis and combining ability for quality and yield in early maturing single cross hybrids of maize(Zea mays L.). Indian J. Agric. Res., 41:210-214.

El-Badawy, M. E. M. (2006). Genetic analysis of diallel crosses in maize (Zea mays L.) over two years. Annals of Agric. sci. Moshtohor, 44(3):911-922.

El-Ghonemy, M.A. and M.H.A. Ibrahim (2010). Diallel analysis of yellow maize for combining ability and heterosis. J. Plant production, Mansoura Univ., 1(6): 779-92. 
El-Hosary, A.A.; Mohamed, M.K.; Sedhom S.A. and Abo-El-Hassan, G.K.A. (1994). Performance and combining ability in diallel crosses of maize. Annals of Agric. Sci. Moshtohor. 32(1) : 203215.

FAO. (2011). Area of maize harvested (ha) in 2009, food supply (kcal/capita/day) and protein supply quantity (g/capita/day) in 2007. Available at http:faostat.fao.org/site/567/Desktop Default. Aspx? Page ID=567\#ancor (verified 7 Jan. 2012). Food and Agric. Organization of the United Nations, Rome, Italy.

Griffing, B. (1956). Concept of general and specific combining ability in relation to diallel crossing system. Australian J. Biol. Sci., 9: 463-493.

Irshad-El-Haq, M., S.U. Ajmal, M. Munir and M. Gulfaraz (2010). Gene action studies of different quantitative traits in maize. Pak. J. Bot., 42: 1021-1030.

Katta,Y.S; M.S.M. Abd El-Aty.; M.A. El-Hity and M.M. Karmara (2007). Estimate of heterosis and combining ability of some white inbred lines of maize (Zea mays L.). J. Agric. Sci .Mansoura Univ., 32(9): 70777088.

Nawar, A. A.; S.A. El-Shamarka and E.A. El-Absawy (2002). Diallel analysis of some agronomic traits of maize. J. Agric. Sci. Mansoura Univ., 27(11): 7203-7213.

Oliveira S.M. (2011). Genetic parameters of maize population in contrasting nitrogen levels. Rev. Cienc. Agron. Vol. 42,n.1,PP. 168174 ISSN 1806-6690.

Raun, W. R. and G. V. Johnson (1999). Improving nitrogen use efficiency for cereal production. Agron. J., 91:357-363.

Sedhom, S. A. (1994). Estimation of general and specific combining ability In maize under two different planting dates. Annals of Agric. Sci. Moshtohor, 32(1) : 119-130.

White, P. J. and L. A. Johnson (2003). Corn: Chemistry and technology. 2nd Ed., American Association of Cereal Chemists, St. Paul, MN., USA., ISBN-13:9781891127335,Pages:892. 
القدرة علي التآلف وقوة الهجين لبعض صفات التزهير والصفات الخضرية لخمسـة

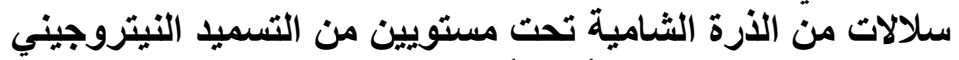

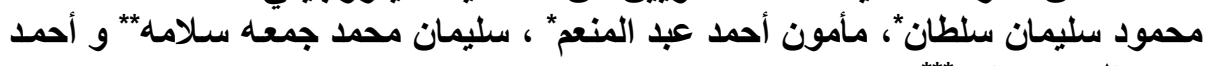

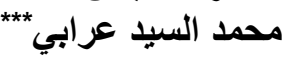

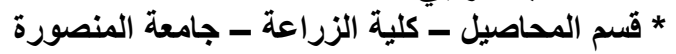

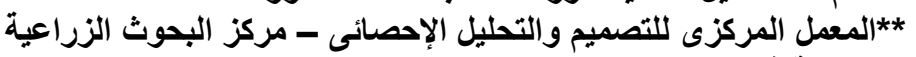
(شثركة فاين سيدز إنترناشيونال

الهدف من البحث التحليل الور اثى لبعض الصفات في بعض سـلالات الذرة الثـامية و الهجن الناتجـة

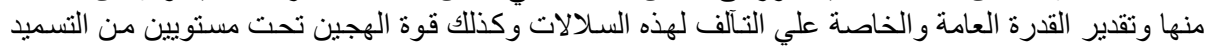

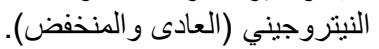

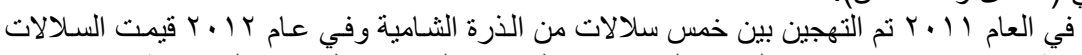

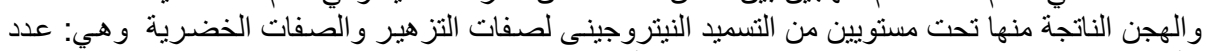

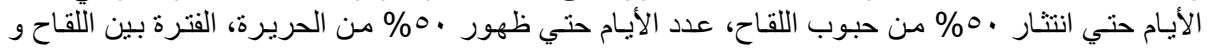

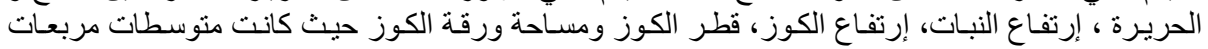

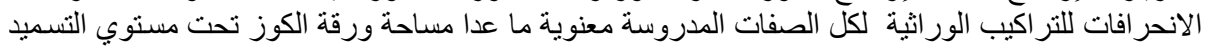

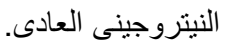

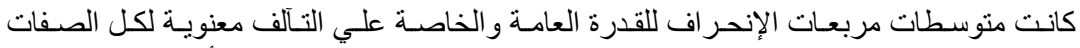

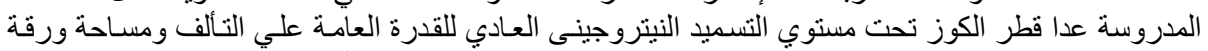

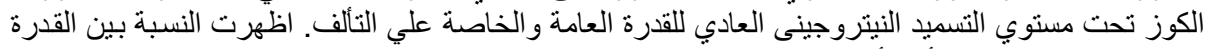

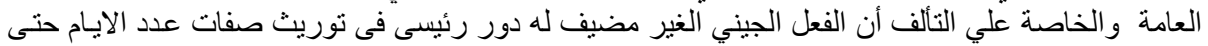

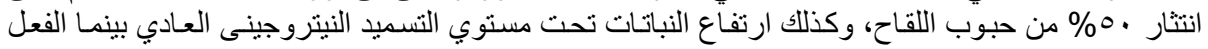

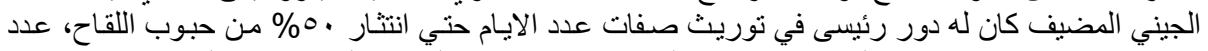

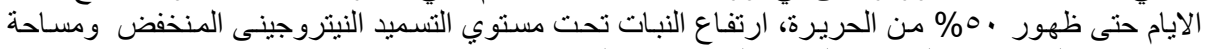

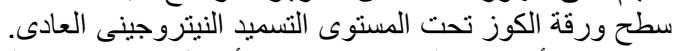

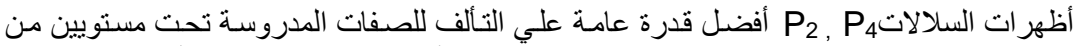

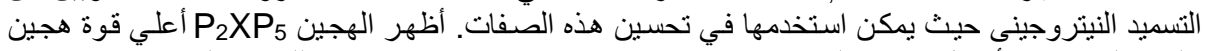

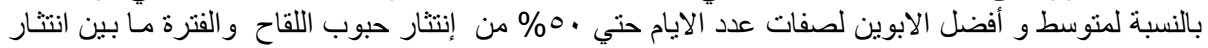

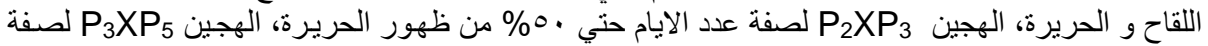

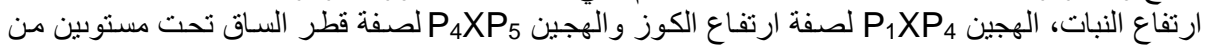

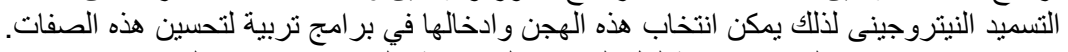

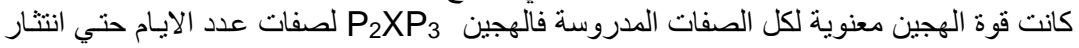

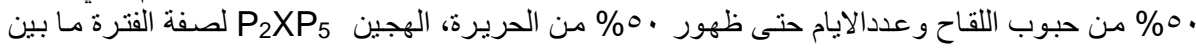

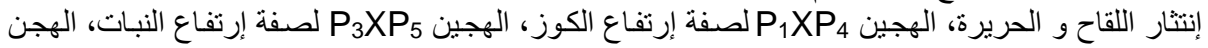

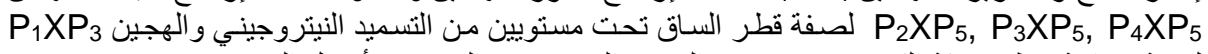
لصفة مساحة سطح ورقة الكوز تحت مستوى التسميد النيتروجيني المنخفض أفضن النض الهن.

كلية الزراعة - جامعة المنصورة كلية الزراعة بمشتهر - جامعة بنها
قام بتحكيم البحث

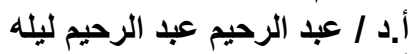

أ.د / محمود الزعبلاوى محمود الرحيل 